\title{
CIL II 5407 Y OTROS EPÍGRAFES DE ASIDO CAESARINA*
}

\author{
Julián González / Salvador Montañés \\ Universidad de Sevilla / Museo Arqueológico de Medina Sidonia \\ julgonzalez@us.es /geha-arqueologia1@hotmail.com
}

\section{CIL II 5407 AND OTHER INSCRIPTIONS FROM ASIDO CAESARINA}

RESUMEN: En los últimos años, fruto del intenso trabajo arqueológico desarrollado en Medina Sidonia por los arqueólogos locales, se han producido nuevos hallazgos epigráficos y el redescubrimiento de algunos epígrafes que se consideraban perdidos, entre ellos el conocidísimo CIL II 5407, que han venido a aumentar el ya rico corpus de Asido Caesarina.

PALABRAS CLAVE: Epigrafía, onomástica, Medina Sidonia, terminus priuatus, graffiti.

\begin{abstract}
In recent years, as a result of the intensive archaeological work developed in Medina Sidonia by local archaeologists, there have been new Epigraphic finds and the rediscovery of some items that were considered lost, among them the well-known CIL II 5407, which have come to increase the already rich corpus of Asido Caesarina.
\end{abstract}

KEYWORDS: Epigraphy, Onomastic, Medina Sidonia, terminus priuatus, graffiti.

RECIBIDO: 09.09.2013. ACEPTADO: 15.01.2014

\section{INSCRIPCIÓN CIL II 5407.}

Hace algunos años, en un vertedero de escombros situado a la entrada de Medina Sidonia, por la carretera de Chiclana de la Frontera, tuvo lugar el redescubrimiento de una de las inscripciones de esta localidad más comentadas y estudiadas y de la que tan sólo teníamos noticias a través de un calco realizado por don Mariano Pardo de Figueroa (Doctor Thebussem) a finales del siglo XIX.

* Este artículo se enmarca dentro de las actividades que el Museo Arqueológico de Medina Sidonia (Cádiz) realiza de potenciación del estudio de sus fondos y colecciones, y como una aportación más al conocimiento de la epigrafía romana gaditana de la que los autores del mismo se vienen ocupando desde hace años. Igualmente agradecemos al Dr. Abascal su amabilidad al leer el original de este trabajo, así como sus siempre sugerentes observaciones. 




Figura 1a. Foto de la columna con detalle situación cartela (Foto, Alberto Ocaña Erdozáin)
Se trata de una columna de una altura de $1,94 \mathrm{~m}$ y un diámetro de $28,49 \mathrm{~cm}$, rematada por un baquetón doble de 3,5 cm y 1,5 $\mathrm{cm}$ de altura, respectivamente, con un orificio de unos $5,5 \mathrm{~cm}$ de diámetro en su parte superior. Las letras son capitales cuadradas, de elegante factura, probablemente de la primera mitad del siglo I d.C.

Pocas inscripciones han sido tan estudiadas y citadas por los estudiosos como ésta, por lo que su recuperación podrá contribuir a aclarar las controversias que todavía hoy existen sobre la información facilitada por dicho epígrafe, especialmente sobre el estatuto de Asido Caesarina.

La primera noticia sobre su hallazgo nos la proporciona don Mariano Pardo de Figueroa en un artículo publicado en 1888, según el propio autor, en un periódico (probablemente en el Diario de Cádiz), indicando que unos diez o doce años antes se encontró en las proximidades de la antigua iglesia visigótica llamada de Los Santos, a un kilómetro aproximadamente al SO de Medina, "una columna o cipo terminal de piedra berroqueña, con altura de 1,92 metro por 0,28 y 0,30 de diámetro en sus respectivas extremidades. Se encuentra hoy en la casa calle Arrieros, núm. 8, propiedad de D. José Butrón. A los 0,75 metros de la parte inferior de dicha piedra aparecen estas seis letras:

\section{D \\ C Cs A $\mathbf{A}^{1}$}

Aureliano Fernández Guerra, Eduardo de Saavedra y el Padre Fita, consultados por el Dr. Thebussem, llegaron a la conclusión

${ }^{1}$ Cfr. Dr. Thebussem, "Tres preguntas. Á Don Adolfo de Castro", en Tercera ración de artículos (Madrid 1898) 385-389. 
de que se trataba de un terminus municipalis que deslindaba su territorio con los de algunas ciudades vecinas, aunque reconocían que "los límites no se determinan sino por el emperador o un juez delegado del César", por lo que con grandes dudas y vacilaciones transcribieron el texto en los siguientes términos:

\section{D(ecurionum) D(ecreto) / C(onuentus) G(aditanus) A(ger) A(sidonensis)}

o, con el cambio del nominativo por el genitivo,

\section{D(ecurionum) D(ecreto) / \\ C(onuentus) G(aditani) A(ger) A(sidonensis)}

Hübner, a través de un calco, recibido igualmente de Mariano Pardo en julio de 1888, transcribió el texto como

\section{D / C G A A}

y aunque rechaza la lectura anterior de Saavedra y Fernández Guerra por ir 'contra usum', no se atreve a dar ninguna transcripción, pues, según su opinión, Asido y Gades serían municipios y no colonias. Insiste en que es probable que se trate de términos, pero, ante las dificultades que plantea la identificación de la colonia, y además porque esperaríamos C(olonorum) C(oloniae), se inclina por considerar que tal vez 'possint etiam indicati esse fines priuati'

La crítica posterior, prácticamente en su totalidad ${ }^{3} \mathrm{y}$ a pesar de haberse perdido la columna y no poder constatar cuál era la lectura correcta, se ha inclinado por corregir la $\mathrm{G}$ por una $\mathrm{C}$ y leer

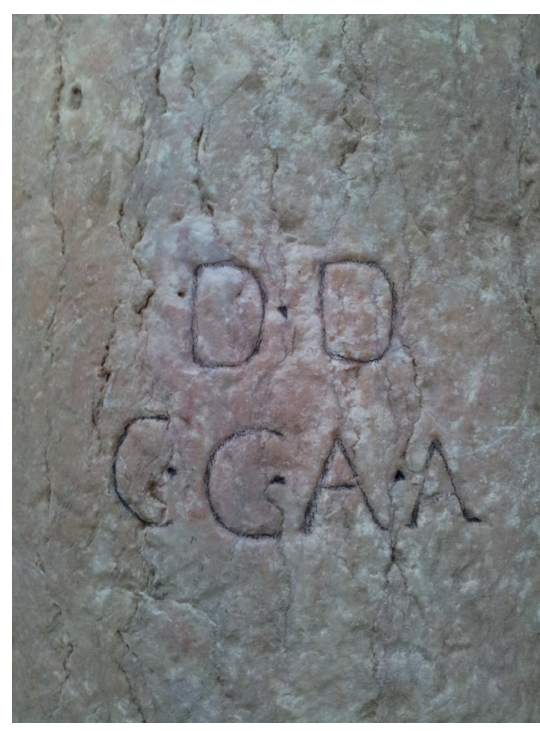

Figura 1b. Detalle del texto (Foto, Alberto Ocaña Erdozáin)

${ }^{2}$ CIL II 5407.

${ }^{3}$ E. Romero de Torres, Catálogo monumental de España. Provincia de Cádiz (Madrid 1934) 216, mantiene la lectura ofrecida por el Dr. Thebussem; J. González, Inscripciones romanas de la provincia de Cádiz (Cádiz 1982) 19-20; ídem, “Asido quae Caesarina”, en J. González, J. C. Saquete (eds.), Colonias de César y Augusto en la Andalucía romana (Roma 2011) 277, prefiere, ante la ausencia de autopsia, no inclinarse por una u otra hipótesis. 


\section{C(olonia) C(aesarina) A(ugusta) A(sidonensis) ${ }^{4}$,}

sin aclarar de forma concluyente, y en muchos casos sin ni siquiera intentarlo, cuál sería la función asignada a dicha inscripción, vacilando entre una columna que sostuvo en su momento una estatua ${ }^{5}$, en cuyo caso la inscripción serviría para indicar que el ofrecimiento se hizo por decreto de los decuriones de Asido, o bien considerar probable que el cipo marcara un límite del territorium de la colonia, en cuyo supuesto el epígrafe debería interpretarse en el sentido de que la colonia es la donante del cipo y del objeto que pudiera sostener, pero no de la limitación de su territorium, cuestión que no pertenecía a las atribuciones de la curia local".

Esta interpretación, en principio verosímil, tropieza con ciertas dificultades surgidas a partir de la titularidad atribuida a la colonia: Caesarina Augusta. En primer lugar, todos los estudiosos ${ }^{7}$ consideran que el epíteto Caesarina apunta con claridad a una fundación realizada por los triúnviros, según los planes de César, al igual que ocurre con su homónima Norba Caesarina. Su status sería muy probablemente el de colonia Latina, transformada por Augusto en una colonia ciuium Romanorum, de acuerdo con su conocido interés por realzar el status de las colonias romanas, que le llevó a menospreciar las comunidades que nunca habían sido colonizadas, aunque gozasen del derecho Latino ${ }^{8}$.

Junto a Caesarina, el epíteto Augusta sería, según estos estudiosos, un claro reflejo de la intervención de Augusto en la colonia, ya sea mediante un beneficium concedido a la misma después del 27 a.C., ya sea debida a un nuevo asentamiento de veteranos 9 , afirmaciones que, como veremos, se muestran inconsistentes. Sin embargo, el título de Augusta atribuido a la colonia no está documentado en fuente alguna y, aunque en términos objetivos su ausencia en las mismas no significa su inexistencia, no deja de ser sorprendente su omisión en la obra de Plinio, sobre todo, si tenemos en cuenta, por un lado, que éste utiliza los escritos de

${ }^{4}$ Cfr. M. I. Henderson, "Iulius Cesar and Latium in Spain”, JRS 32 (1942) 13; F. Vittinghoff, Römische Kolonisation und Bürgerrechspolitik unter Caesar und Augustus (Wiesbaden 1951) 104; A. García Bellido, "Las colonias romanas de Hispania", AHDE 29 (1959) 476; H. Galsterer. Untersuchungen zum römischen Städtewesen auf der iberischen Halbinsen (Berlin 1971) 20, n. 34; A. Tovar, Iberische Landeskunde. I. Baetica (Baden-Baden 1974-76) 150; A. Padilla Monge, “Asido Caesarina: consideraciones acerca de su 'status'", Habis 16 (1985) 307-327.

${ }^{5}$ La posibilidad de que la columna hubiese tenido una pequeña estatua encastrada en dicho orificio, que habría sido sustituida por el capitel en un momento posterior, resulta muy improbable, dadas las dimensiones de la columna, casi $2 \mathrm{~m}$ de altura, y del diámetro de su parte superior, apenas $30 \mathrm{~cm}$, lo que produciría un efecto estético anormal, y además los ejemplos de pedestales cilíndricos que conocemos apenas si rebasan el metro de altura y tienen un diámetro de más o menos $60 \mathrm{~cm}$.

${ }^{6}$ Así, por ejemplo, Padilla, op. cit. 326.

${ }^{7}$ Excepto P.A. Brunt, Italian Manpower 225 B.C.-14 A.D. (Oxford 1971) 235, que opina que muy posiblemente pudiera deberse a Octaviano.

${ }^{8}$ Cfr. González, “Asido quae Caesarina”, 279-281.

${ }^{9}$ Cfr. Brunt, op. cit. 235. 
Agrippa y las formulae prouinciarum que habitualmente se fechan en torno al 6 a.C., es decir, en un momento posterior a la fundación de ésta, que se sitúa en torno al 15/13 a.C., y por otro, que todas las colonias augusteas, cuyas deductiones se piensa tuvieron lugar en estos mismos años con motivo de la gran reestructuración administrativa de Hispania realizada por Augusto, sí muestran el título de Augustas en la obra de Plinio.

Por otra parte, sabemos por las fuentes literarias y epigráficas que las sucesivas intervenciones de Augusto en las fundaciones cesarianas no modifican el título de las mismas, y así, aunque sabemos que se han producido nuevas deductiones de veteranos de Augusto en colonias cesarianas, con su evidente reflejo en su adscripción a la tribu Galeria, que convive con la Sergia propia de la fundaciones de aquél, éstas siguen llamándose colonia Patricia, colonia Romula, colonia Genetiua, colonia Regia, etc., sin que en ningún caso se haya añadido el título de Augusta. Resulta, pues, evidente el interés de Augusto en respetar por completo las primitivas denominaciones de las fundaciones debidas a la intervención indirecta de su padre.

Además, en el supuesto de que la iniciativa hubiese partido de la propia ciudad, en respuesta a algún beneficio recibido de aquél, no podemos olvidar que el procedimiento usual en estos casos consistía en enviar una embajada a Roma a solicitar del emperador la aceptación de tal privilegio, según sabemos con certeza por la epistula de Vespasiano dirigida a los decuriones y cuatórviros de $\mathrm{Sa}$ $b o r a^{10}$. No resulta, pues, aventurado vaticinar que Augusto habría rechazado un honor que implicaría una modificación en la titulación de la colonia con la consiguiente intromisión en la memoria y el honor de su padre.

El reciente hallazgo de la pieza ha venido a confirmar que la intuición de los estudiosos era acertada y que se trata ciertamente de dos C, ya que tanto la Gs del Dr. Thebussem, como la G de Hübner, son en realidad una grieta en la piedra, por lo que en un principio pudiéramos pensar que la lectura propuesta $C(O-$ loniae) C(aesarinae) A(ugustae) A(sidonensis) es igualmente la correcta, pero, como ya hemos indicado, los datos no van en esa dirección. En nuestro intento de aclarar el motivo por el que este epígrafe ha sido grabado requiere determinar, en primer lugar, si se trata de una inscripción honoraria o de un término territorial, público o privado, $\mathrm{y}$, en segundo, precisar qué desarrollo hemos de dar a estas abreviaturas.

Hübner, según la información facilitada por el Dr. Thebussem, pues no llegó a ver el original, dice que se trata de un "cippus cylindri forma sive columna cum capite", es decir, una columna con su capitel, que muy probablemente habría estado encastrado en el orificio mencionado.

${ }^{10} C I L \mathrm{II}^{2} / 5,871=C I L$ II 1423 (p. 867, 1049) = ILS 6092. 
Otro dato a tener en cuenta a la hora de valorar la función del epígrafe es su hallazgo, según el explícito testimonio del Dr. Thebussem, no en un ámbito forense, como esperaríamos tratándose de un epígrafe dedicado por el ordo de la colonia, sino extra muros de la colonia, en el entorno de la ermita de Los Santos, asentada sobre el solar de una villa romana, como la mayoría de las fundaciones de este tipo en la zona. Por último, hemos de señalar la ausencia del nombre del homenajeado, los motivos de dicho honor y el objeto dedicado, elementos todos característicos de los epígrafes honorarios.

Todos estos datos apuntan con claridad a que no puede tratarse de una inscripción honoraria, sino que, al contrario, tendríamos una inscripción de aplicación en un ámbito privado, pese a su grabación con motivo de un decretum decurionum, con cuyo enfoque hemos de intentar encontrar una respuesta a la función asignada a la misma por el autor del epígrafe.

Descartada su condición de inscripción honoraria, pensamos que muy probablemente se trate de un epígrafe cuya finalidad es señalar, como ya supuso Hübner, los límites de unos terrenos privados "praesertim cum possint etiam indicati esse fines privati", pues los litigios entre ciudades o entre ciudades y particulares sobre la propiedad del territorio se solventaban con la intervención del gobernador provincial ex auctoritate principis, y no mediante un decreto de los decuriones.

A pesar de que los agrimensores nos advierten de que los conflictos entre particulares y ciudades eran frecuentes, especialmente en África ${ }^{11}$, no tenemos ningún hito de estas características ni en Hispania ni en todo el Occidente, aunque sí algunos ejemplos en África, concretamente uno del África proconsularis, entre la tribu de los Musulami y Valeria Atticilla ${ }^{12}$; y dos de la Mauritania Caesarensis, entre la ciudad Regia y el saltus $\mathrm{Cu}[---]^{13}$, y la tribu de los Tabianenses y el veterano Surus ${ }^{14}$, respectivamente.

\footnotetext{
${ }^{11}$ Agenn. 84,29-85,4 L = Th. 45.16-22: inter res publicas et priuatos non facile tales in Italia controuersiae mouentur, sed frequenter in prouinciis, praecipue in Africa, ubi saltus non minores habent priuati quam res p. territoria, quin immo multi saltus longe maiores sunt territoriis; habent autem in saltibus priuatis non exiguum populum plebeium et uicos circa uillam in modo municipiorum. Cfr. M. J. Castillo Pascual, Espacio en orden: el modelo gromático-romano de ordenación del territorio (Logroño 1996) 184-185; B. Campbell, The writings of the roman land surveyors. Introduction, text, translation and commentary (London 2000) 43, 349 n. 56.

${ }^{12}$ AE 1923, 26: Ex auctoritate / imp. Neruae Traiani Caesaris / Augusti Germanici Dacici pontif. / maximi trib. potest. VIIII imp. IIII cos. V p.p. / L. Minicius Natalis leg. Aug. pr. pr. inter Mu-/sulamos et Valeriam Atticillam / LXXXX a(d) p(roximum) p(assus) CXVI(milia)CD.

${ }^{13}$ AE 1895, 68, CIL VIII, 21663; ILS 5963: Ex au[ctor]itate / Imp. Caes. diui Traiani Parthici [f.] / diui Neruae nepotis Traian[i] / Hadriani Aug. p.p. p.m. tr.pot. XX[I] / cos. III procos. auspiciis L. Aeli C[a-lesaris] Imp. \{Imp \} fil. cos. termini pos(iti) i[n-/ter Regienses et saltum Cu[---] / per C. Petronium Celerem proc. Au[g.] / an(no) prouin(ciae) LXXXXVIII.

${ }^{14}$ AE 1975, 952: Terminum i-/nter Tabia-/neses et Suru-/m ueter\{e\}an-/um.
} 
Sea cual sea la razón de esta escasez de documentos, hay que destacar que en todos los ejemplos de litigios entre comunidades y particulares es necesaria la intervención del gobernador provincial en representación de la autoridad imperial, situación que no se produce en nuestro epígrafe, donde la autoridad radica en el ordo decurionum, por lo que hemos de llegar a la conclusión de que posiblemente se trate o bien de un pleito planteado en el seno de la propia colonia entre la misma y algún propietario, vecino de la misma, que haya ocupado el ager publicus, o bien de un litigio entre particulares intra fines coloniae, materias que caían dentro del ámbito competencial de los magistrados de la colonia Caesarina, según el explícito testimonio de las leyes coloniales y municipales y el testimonio de los gromatici. Aunque no podamos establecer a priori si los terrenos colindantes eran públicos o privados, es decir, si limitaban con el territorio de la colonia o con otra finca particular, nos inclinamos por la primera posibilidad.

El carácter público de los limites y de las uiae está ya reconocido en la lex agraria del 111 a.C. ${ }^{15}$, y la prohibición específica de que nadie construya, are, obstruya o intercepte los limites decumanique y las fossae limitales lo es en el cap. IV de la lex Mamilia Roscia Peducaea Fabia del 55 a.C. ${ }^{16}$. El texto de la lex Mamilia aparece reflejado casi literalmente en el cap. CIIII de la lex coloniae Genetiuae Iuliae ${ }^{17}$.

Pero sin duda el dato más relevante para nuestra empresa está contenido en el cap. V de la misma lex Mamilia, en el que se recoge que nadie arranque o desplace de su emplazamiento los termini colocados por el deductor de la colonia, y que tengan jurisdicción sobre el asunto los magistrados de la colonia ${ }^{18}$.

La misma preocupación por la estabilidad de los limites territoriales está presente en las leyes municipales, así, por ejemplo, la lex Flauia municipalis en su capítulo LXXVI autoriza a los duóviros, conforme a un decreto de los decuriones, a visitar e inspeccionar las lindes, los campos privados y los terrenos

${ }^{15}$ CIL I $\mathrm{I}^{2}$ 585; Riccobono, FIRA $\mathrm{I}^{2}$, Leges, p. 120: quei [ager in Africa est... quae uiae in eo] agro ante quam Carthago capta est, fuerunt: eae omnes publicae sunto limitesque inter centuria[s].

${ }^{16}$ Riccobono, FIRA I², Leges, p. 139: KL IIII: Qui limites decumanique hac lege deducti erunt, quaecumque fossae limitales in eo agro erunt, quaecumque fossae limitales in eo agro erunt, qui ager hac lege datus adsignatus erit, ne quis eos limites decumanosque obsaeptos neue quid in eis molitum neue quid ibi obsaeptum habeto, neue eos arato, neue eis fossas opturato neue obsaepito, quominus suo itinere aqua ire fluere possit. Si quis aduersus ea quid fecerit, is in res singulas, quotienscumque fecerit, HS IIII(milia) colonis municipibusue eis, in quorum agro id factum erit, dare damnas esto.

${ }^{17}$ CIL I 594; CIL II ${ }^{2} / 5,1022 ; C I L$ II $5439=$ ILS 6087; CILA II, 611.

${ }^{18}$ Riccobono, FIRA I ${ }^{2}$, Leges, p. 140: KL V: Qui hac lege coloniam deduxerit... limites decumanique ut fiant terminique statuantur curato... Quique termini hac lege statuti erunt, ne quis eorum quem eicito neue loco moueto sciens dolo malo. Si quis aduersus ea fecerit, is in terminos singulos, quos eiecerit locoue mouerit sciens dolo malo, HS V m(ilia) n(ummum) in publicum eorum, quorum ??? fines is ager erit, dare damnas esto; deque ea re curatoris, qui hac lege erit, iuris dictio... esto. Cum curator hac lege non erit, tum quicumque magistratus in ea colonia... iure dicundo ???, eius magistratus de ea re iurisdictio iudicisque datio addictio esto... 
públicos arrendados ${ }^{19}$, sin duda para comprobar que los límites no han sido modificados por la intervención de particulares.

También los gromatici señalan la existencia de controversias entre colonias y municipios con particulares sobre la propiedad de determinados lugares de propiedad pública que habían sido ocupados arbitrariamente por alguno de ellos. Esto ocurre especialmente con los terrenos que habían quedado sin asignar a los colonos, los llamados subseciua, acerca de los cuales nos cuenta Agennius Vrbicus que "los propietarios de las tierras vecinas invadieron y ocuparon las tierras desocupadas, y como si la disponibilidad de la tierra desocupada los hubiese incitado, durante un largo período de tiempo las unieron a su propiedad con impunidad"20, añadiendo que sobre estas controversias las competencias correspondían a las comunidades ${ }^{21}$.

Por otra parte, la adsignatio en una colonia se extiende hasta donde la tierra es cultivable y termina en el punto donde la formación de las centurias se interrumpió, pero más allá existían generalmente bosques y montañas, que proporcionaban un límite indiscutible a la colonia. Estos territorios, propiedad de la colonia, pero que no habían sido asignados, fueron a menudo ocupados por los propietarios vecinos y, atraídos por la oportunidad, se apropiaron de la tierra. Contra estos actos existía también la jurisdicción de la colonia ${ }^{22}$.

Así, pues, un decreto del ordo decurionum pondría fin a una disputa sobre la propiedad de unas tierras entre dos propietarios privados o, lo que es más frecuente, entre la propia colonia y un habitante de la misma, que hubiese invadido y usurpado unos terrenos de propiedad pública.

Ahora bien, si los testimonios de hitos que pongan fin a litigios entre ciudades y entre ciudades e individuos ajenos a las mismas son escasos, aún lo son mucho más en el caso de propiedades privadas, hasta el punto de que tan sólo hemos encontrado dos referencias a un decretum decurionum, una en una inscripción de la Galia Cisalpina, aunque no se trata de una terminatio, sino de la

${ }^{19}$ Cfr. J. González, “The lex Irnitana: a new copy of the Flavian municipal law”, JRS 76 (1986) 147 ss = CILA II,4, pp.184-85: duumuir municipi Flaui Irnitani suo quisque anno ad decuriones conscriptosue eius municipi, cum eorum partes non minus quam duae tertiae aderunt, referto fines agros uectigalia eius municipi eo anno circumiri recognosci placeat, deque ea re facito uti decurionum conscriptorumue decretum hac lege fiat.

${ }^{20}$ Agenn. p. 81,7 = 53,16 L; 40,24 Th: per longum enim tempus attingui possessores uacantia loca quasi incitante otiosi $<$ loci> opportunitate inuaserunt et per longum tempus inpune commalleauerunt.

${ }^{21}$ Agenn. p. 81,7 = 53,26; 40,24 Th: ideoque semper hoc genus controuersiae a rebus publicis exercentur. Cfr. Campbell, op.cit. 38-39.

${ }^{22}$ Agenn. p. 86,10 =55,8; 47,2 Th: adsignatio peruenit usque qua cultum fuit, quatenus ordinatione centuriarum intermissa finitur. Vltra autem siluestria fere fuerunt et iuga quaedam montium, quae uisa sunt finem coloniae non sine magno argumento facere posse... haec plerumque proximi possessores inuadunt et opportunitate loci inuitati agrum optinent. Cum his controuersiae a rebus publicis solent moueri. Cfr. Campbell, op. cit. 44-45. 
mención de un cambio en la posición del inicio de un $\operatorname{limes}^{23}$, y dos, en un epígrafe procedente del propio conuentus Hispalensis, concretamente de Arcos de la Frontera, que, en nuestra opinión, ayudará a disipar todas las dudas surgidas al respecto, y cuyo texto es el siguiente:

\section{D. \\ Q. C. Q.}

Las primeras noticias sobre esta inscripción proceden de una obra manuscrita del erudito Juan Camacho del Real de finales del siglo XVIII, en la que comenta que "también se descubre en uno de los ángulos exteriores del nuevo templo del Arcángel San Miguel otro monumento sobre una columna que dice así: D.D. / Q.C.Q.”. Un siglo después, en 1896, el doctor Miguel Mancheño y Olivares intentó en vano encontrar el epígrafe en cuestión, y así nos dice que "en efecto, en el ángulo exterior izquierdo de la mencionada iglesia, hemos encontrado después de quitar una pared de ladrillo que la cubría, una base de columna, pero ni existe ésta ni la inscripción citada por Camacho"24.

El paralelismo entre ambos epígrafes es indiscutible y resulta un tanto sorprendente que los estudiosos que se han ocupado de desarrollar las abreviaturas C. C. A. A. de nuestra inscripción no hayan reparado en esta semejanza, que incluso se extiende al tipo de soporte, una columna.

Es evidente que detrás de las abreviaturas Q. C. Q. se ocultan los tria nomina de un propietario privado, al que le ha sido asignado el límite de su propiedad como consecuencia de un litigio solucionado mediante ese decreto de los decuriones, ya que la indicación del nombre del propietario mediante simples abreviaturas es la norma habitual, según el testimonio de los escasos termini conocidos con indicación de propiedades privadas. Sirva de ejemplo uno encontrado en noviembre del 2000, en el Africa proconsularis en la localidad de Henchir Ben Khelil, a 2 km de Henchir el Begar (Casae Beguenses), con los nombres de los propietarios abreviados:

\section{Q. A(nici) F(austi) / b) C. M(---) P(----25}

No deja de resultar atractiva la idea de identificar a este anónimo propietario con Q(uintus) Calpurnius, atestiguado en el epígrafe de Calpurnia Q.f. Galla,

${ }^{23}$ CIL V 2456 = ILS 5986: Caput limitis / Lontriconis / permutatum / ex d.d.

${ }^{24}$ Juan Camacho del Real, Memorias compendiadas de las Antigüedades de Arcos, cap. IV, ms. 1790; Miguel Mancheño y Olivares, Apuntes para una historia de Arcos de la Frontera (Arcos 1896) 631; J. González, Inscripciones romanas de la provincia de Cádiz (Cádiz 1982) 515.

${ }^{25}$ AE 2003, 1974. 
que ha recibido el honor de una estatua en virtud de un decreto de los decuriones y del pueblo ${ }^{26}$.

De acuerdo a cuanto venimos diciendo en la páginas precedentes, nuestro cipo señalaría el limes entre unos terrenos de propiedad pública, muy probablemente unos subcesiua, y una propiedad privada, y habría sido colocado en virtud de un decreto de los decuriones de la colonia para poner fin a un litigio surgido por la invasión que este propietario habría realizado sobre dicho ager publicus. Este supuesto resulta más congruente con la finalidad última de este tipo de pleitos: señalar los límites de las propiedades privadas. Esta hipótesis tiene, como es evidente, el problema de las cuatro abreviaturas en lugar de los tria nomina habituales, pero el añadido de un segundo cognomen o incluso la indicación de la origo resulta bastante corriente y no debería invalidar, al menos de forma concluyente, la viabilidad de la misma. Por todo ello, consideramos muy probable el siguiente desarrollo de las abreviaturas:

\section{G(ai) C(---) A(---) A(sidonensis) ${ }^{27}$}

No faltan en Asido gentes cuyos gentilicios empiecen por C-: Cassii, Cornelii, Claudii, Clodii, e, incluso, están documentados un liberto, C. Cassius Pylades, y un joven de 18 años, C. Clodius C.f. Gal. Blattianus, en cuyo honor el ordo ha decretado diversas honras fúnebres: locum sepulturae, turis p.c., statuam, y que sin duda pertenece a una distinguida familia de la colonia, que tienen G(aius) como praenomen. Sin embargo, hemos preferido no intentar desarrollar la abreviatura del nomen.

\section{InSCRIPCIÓN DE $Q$. CORNELIVS MATERNVS}

Placa de mármol blanco, en buen estado de conservación, pues sólo ha perdido un pequeño fragmento en su ángulo superior derecho y algunas lascas en su perímetro. La parte posterior presenta un engrosamiento del mármol ocupando casi toda la mitad superior $(11 \mathrm{~cm}$ de alto por todo el ancho de la placa, y un grosor que oscila entre los $5,8 \mathrm{~cm}$ de la parte central hasta los aproximadamente 3,3 $\mathrm{cm}$ de los extremos), no alisado y con signo de roturas antiguas y alguna reciente; mientras que la mitad inferior aparece perfectamente alisada $(13 \mathrm{~cm}$ de alto, 30 $\mathrm{cm}$ de ancho y grosor uniforme de 2,6 cm). Los cantos superior e inferior están bien cortados y pulidos, mientras que el izquierdo y derecho son irregulares, sin dar la impresión de rotura, más bien no acabados. Mide $24 \mathrm{~cm}$ de altura, $30 \mathrm{~cm}$ de anchura; la altura de las letras oscila entre $4,1 \mathrm{~cm}$ de la 1.1 y los $3,7 / 3,8$ de

\footnotetext{
${ }^{26}$ CIL II 1364 = Romero de Torres, op.cit. 181 = González 1982, 514: Calpurniae Q.f. / Gallae d.d. / et populi Clodia C.f. / Optata mater honore / usa inpensam remisit.

${ }^{27}$ No se puede descartar la posibilidad de que en la segunda A haya que leer A(gri), es decir, la inscripción haría referencia a que estos campos son propiedad de G C A.
} 


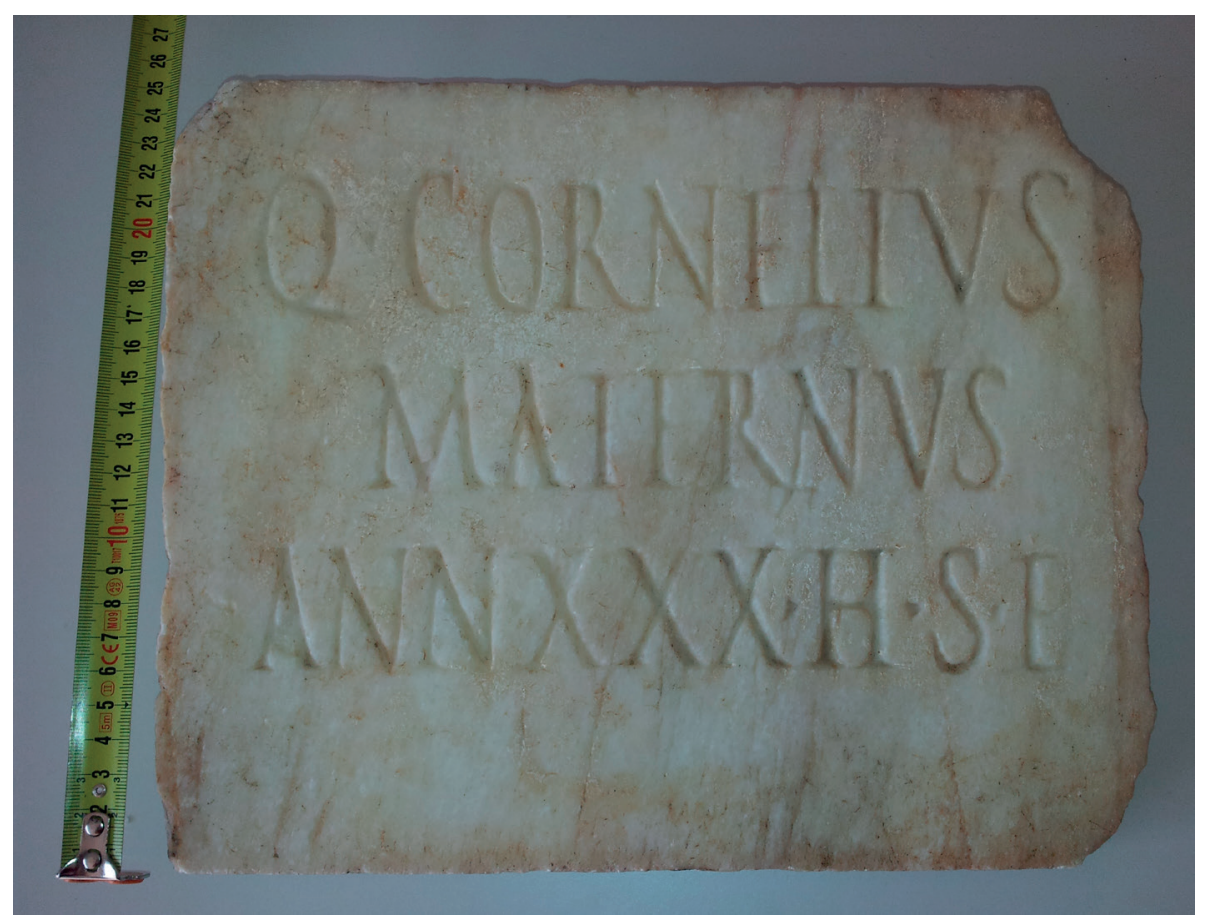

Figura 2. Inscripción de M. Cornelius Maternus.

las 11.2 y 3; la C y la $\mathrm{S}$ de "CORNELIVS" tienen una altura de 4,6 cm. Los puntos son triangulares. Se encontraba en Medina en una casa, según el Padre Jerónimo de la Concepción; posteriormente se perdió, y recientemente, en los años ochenta, fue localizada casualmente en la ladera Oeste del Cerro del Castillo. En la actualidad se encuentra depositada en el Museo Arqueológico de Medina Sidonia. Su texto es el siguiente:

\section{Q(uintus).CORNELIVS \\ MATERNVS \\ ANN(orum).XXX.H(ic).s(itus).E(st)}

"Quinto Cornelio Materno, de 30 años, aquí está enterrado".

Bibliografía: CIL II 1322; Romero de Torres, op.cit. 215; ILER 2548; IRPC 11.

Las letras son capitales cuadradas algo estilizadas, la A es estrecha y el trazo izquierdo no se une con el derecho en su remate e igual ocurre con la $\mathrm{N}$; los trazos de la $\mathrm{E}$ son cortos e inclinados hacia arriba, los senos de la $\mathrm{S}$ amplios, los trazos horizontales cortos y poco marcados, el ojo de la R cerrado, etc. La paginación está muy conseguida mediante una alineación correcta de los márgenes laterales 
y con la 1.2 centrada; algunas letras, como la $\mathrm{C}$ y la $\mathrm{S}$ final del nomen superan ligeramente la línea de la caja. Llama la atención la ausencia de las fórmulas rituales D.M.S. y S.T.T.L.

Los Cornelii constituyen una de los gentilicios más frecuente en Hispania ocupando con más de 500 testimonios el tercer lugar ${ }^{28}$, como un ejemplo, sólo en la provincia de Cádiz hay más de una veintena, entre los que sobresalen las familias senatoriales de los Cornelii Balbi de Gades y los Cornelii Pusiones de El Portal. El cognomen Maternus es también uno de los más frecuentes en Hispania, concretamente el sexto con más de 150 ejemplos ${ }^{29}$.

Fecha: se podría fechar este epígrafe en la primera mitad del siglo II d.C. según el tipo de letra y la cuidadosa ordenación del texto.

\section{INSCRIPCIÓN DE VINVLEIA SEVERA}

Placa de mármol blanco que conserva los márgenes izquierdo e inferior y parte del derecho en su parte superior, con ligera inclinación; al parecer el texto estaba enmarcado en el margen derecho por una serie de engrosamientos, de los que se han conservado varios, entre los que existen algunas roturas, y su parte posterior está perfectamente alisada, con pulido irregular. Mide $22 \mathrm{~cm}$ de altura, su anchura oscila entre 17,3 y $19,5 \mathrm{~cm}$ y $2,2 \mathrm{~cm}$ de grosor; la altura de las letras oscila entre los 2,8-3,2 cm; los puntos son pequeñas hederae. Fue encontrada en el transcurso de unas obras en c/ Barrionuevo, al norte de la ciudad actual, salida hacia Jerez de la Frontera a finales de los años setenta, se trata de una zona de necrópolis, si bien hasta ahora sólo se ha excavado un sector con incineraciones del siglo I a.C. En la actualidad se encuentra depositada en el Museo Arqueológico de Medina Sidonia. Su texto es el siguiente:

D(is).M(anibus).S(acrum).

VINVLE-

IA.SEVE-

RA.ANNO

.M(enses).VI.S(it).T(erra).L(euis).

"Consagrado a los dioses Manes. Vinuleya Severa, de 1 año y 6 meses.

Sea (para ti) la tierra leve".

La paginación del texto resulta desordenada, y si la alineación izquierda está bastante conseguida, aunque las 11.1 y 4 se apartan ligeramente de la misma, la

${ }^{28}$ Cfr. J. M. Abascal, Los nombres personales en las inscripciones latinas de Hispania (Madrid 1994) 116.

${ }^{29}$ Cfr. Abascal, op.cit. 418-19. 
Figura 3. Inscripción de Vinuleia Seuera



derecha es muy caótica, pues tan sólo las 11. 1-2 están algo centradas. Las letras son capitales actuarias, de dibujo tosco e irregular con acusados remates superiores, los ángulos de la A, N y M no se forman en el remate de los trazos inclinados sino por debajo del mismo, los trazos horizontales de la A, E y L son cortos e inclinados hacia arriba, la $\mathrm{O}$ y la $\mathrm{V}$ ligeramente ovaladas, etc. Hay que anotar además, por un lado, la puntuación irregular al final de la 1.1 y al principio y final de la 1. 4, y por otro, las omisiones de la fórmula ritual H.S.E. y de la primera $\mathrm{T}$ en S.T.L.

En el numeral ANNO hay que señalar por un lado, la pérdida de la $-m$ final, dado que la nasal en final de palabra apenas se pronunciaba, y por otro, el cambio de $-u$ final secundaria en $-o$, según fenómenos fonéticos característicos del latín hablado de época tardía ${ }^{30}$.

Vinuleia es un gentilicio con muy poco ejemplos en Hispania, apenas cuatro testimonios ${ }^{31}$, uno de ellos, M. Valerius Romulus Timotheus Vinuleianus Vinuleius Gallus, sin duda un destacado personaje, procedente de Barbate (Cádiz), asiento de la antigua Baesippo ${ }^{32}$.

Fecha: siglo III por el tipo de letra, la estructura descuidada del texto y las anomalías léxicas.

\footnotetext{
${ }^{30}$ Cfr. M. Bassols de Climent, Fonética Latina (Madrid 1962) 190-91.

${ }^{31}$ Cfr. Abascal, op. cit. 248.

${ }^{32}$ CIL II 1924; Romero de Torres, op.cit. 246; ILER 6140, 6431; IRPC 70.
} 


\section{InSCRIPCIÓN DE POMPEIA VRBANA?}

Placa de mármol gris con vetas oscuras (le falta un tono más verdoso para identificarlo con claridad como cipollino), que ha perdido en mayor o menor medida sus márgenes en todo el perímetro, mide $12 \mathrm{~cm}$ de altura, $12 \mathrm{~cm}$ de anchura en la base y $11 \mathrm{~cm}$ en la parte superior, y $3,6 \mathrm{~cm}$ de grosor; la altura de las letras oscila entre 2,3-2,5 cm. Fue encontrada hace unas décadas de forma casual entre los cascotes de una pequeña edificación arruinada, en la salida del núcleo urbano situada al sur, en la base de un pequeño cerro donde se localizan enterramientos de incineración romanos, y en su perímetro inhumaciones tardías. El texto de la inscripción es el siguiente:

POMP[EIA.CN.F.?]

VRBA[NA.VXOR?]

AMANT[ISSIMA]

- - - - -

"Pompeya, ¿hija de Gneo?, Urbana, esposa amantísima...".

La parte conservada del texto muestra una alineación correcta, con la 1.2 ligeramente centrada; las letras son capitales cuadradas, de grabado profundo, la A, M y N presentan ángulos cerrados en sus vértices, los ojos de la P, B y R abiertos, el trazo inclinado de la $\mathrm{R}$ prolongado con un ligera curvatura, los trazos de la $\mathrm{M}$ paralelos, la $\mathrm{O}$ redondeada, etc.

La restitución del nomen de la difunta es evidentemente hipotético, pues podría ser Pomponia, pues ambos están atestiguados en la provincia de Cádiz, aunque el primero es mucho más corriente, e igual ocurre con la filiación, que podría haberse omitido, o incluso con el parentesco, ya que podría ser filia, soror, etc., en vez de uxor. Hemos supuesto un nombre femenino por la falta de praenomen. Vrbana es cognomen bastante frecuente en Hispania, con más de 30 testimonios, de ellos dos en Gades $^{33}$.

Fecha: primera mitad del siglo II d.C. por el tipo de letra y el empleo del superlativo amantissima .

${ }^{33}$. Cfr. Abascal, op. cit. 550. 


\section{INSCRIPCIÓN HONORARIA}

Fragmento de placa de mármol blanco, pulido por su parte posterior, que ha perdido todos sus márgenes y adopta una forma rectangular, conservando tan sólo una L completa (1. 2) y la parte inferior de una R (1. 1). Mide $19 \mathrm{~cm}$ de altura, $13,5 \mathrm{~cm}$ de anchura y 4,5 $\mathrm{cm}$ de grosor; la altura de la $\mathrm{L}$ es de $8 \mathrm{~cm}$, la altura de la parte conservada de la $\mathrm{R}$ es de $4 \mathrm{~cm}$ ). Fue encontrada en agosto de 2007 en el transcurso de la segunda campaña de excavación en el castillo de Medina Sidonia, en un nivel medieval, junto a sillares derruidos del castellum romano. $\mathrm{Su}$ texto es el siguiente:

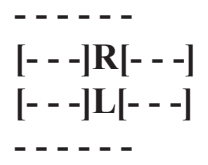

Las letras son capitales cuadradas, de espléndida factura, grabadas a bisel, por lo que se trata sin duda de una inscripción honoraria. Aunque la restitución del texto resulta imposible, quién no se atrevería a pensar, dadas la per-

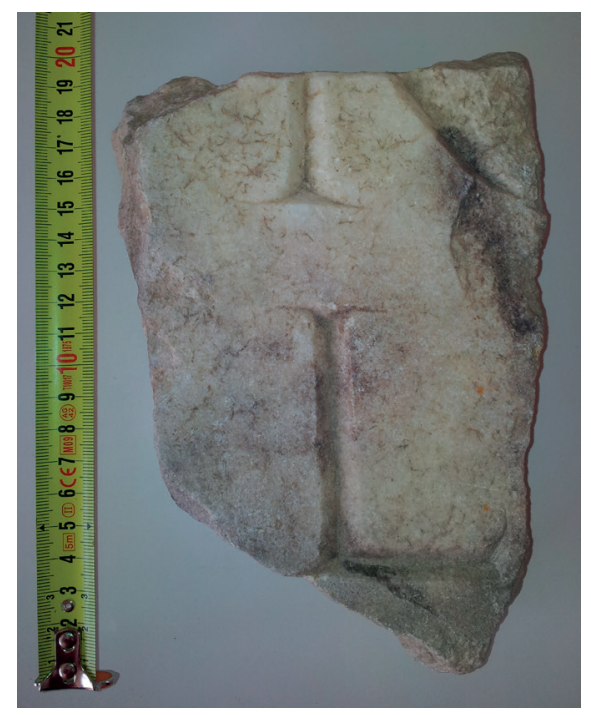

Figura 5. Inscripción honoraria. fección de su ejecución y el tamaño de las letras, en un epígrafe imperial, perteneciendo la R a CAESAR(i) y la L o bien a VLPIO o bien a AELIO.

Fecha: primera mitad del siglo II d.C. según el tipo de letra.

\section{6. ¿INSCRIPCIÓN FUNERARIA?}

Sillar de mármol rosa, que se rompe con desprendimiento de escamas; presenta una forma irregular, pues los laterales derecho e izquierdo son roturas, así como el canto, de $4 \mathrm{~cm}$ de longitud, que coincide en la fotografía con la sigla, está pulido, por lo que nos encontremos con un bloque grueso de mármol de forma irregular, al no encontrarse en paralelo este borde con el frontal donde se encuentra el epígrafe; tiene una profundidad máxima de $12 \mathrm{~cm}$. La parte superior e inferior están pulidas. En la superficie de la parte superior más cercana al borde frontal hay rotura, que afecta levemente a la parte alta de las letras (a la Q). El campo epigráfico tiene unas medidas de $6,3 \mathrm{~cm}$ de alto y $9,3 \mathrm{~cm}$ de anchura; la altura de las letras es más o menos de 3,5 cm. Fue encontrado en el año 1996, en el transcurso de una intervención urbana preventiva en un solar de la C/ Santo Cristo $n^{\circ} 3$, al sur de la ciudad, en el entorno de los restos de una cisterna romana. Su texto es el siguiente: 


\section{$[---] . F . Q .[---]$}

“... hijo de...Quintilio/a...”.

Las letras son capitales cuadradas, de expléndida factura, el trazo inferior de la $\mathrm{F}$ un poco más corto que el superior, la $\mathrm{Q}$ redondeada. Aunque en la parte inferior izquierda ha saltado una lasca, no hay duda que la primera letra conservada es una $\mathrm{F}$.

El hecho de presentar sus caras superior e inferior pulidas (desconocemos si también lo estaban las laterales) nos inclinan a pensar en un monumento, probablemente funerario, con la inscripción grabada a lo largo de una serie de sillares, pues la escasez de texto conservado nos impide pensar que se tratase de uno solo.

La posición de la $\mathrm{Q}$ inicial nos revela que nos indica o la tribu Quirina o un cognomen, lo que nos parece más sugerente, teniendo en cuenta que la tribu de la colonia Caesarina era la Galeria. Aunque los cognomina iniciados con Q- son numerosos, hemos preferido Quintillus por estar ya documentado en la colonia: Flauia Quintilla ${ }^{34}$.

Fecha: siglo II d.C., según el tipo de letra.

\section{INSCRIPCIÓN DE LiCINIA M. LIB. GALLVLA}

Ara de piedra caliza grisácea, que ha sufrido fuerte desgaste por efecto de los agentes atmosféricos, con molduras de base y coronamiento, sobre el que se encuentra el frontón, decorado con dos rosetas de cinco pétalos y botón central y que tiene en los extremos dos puluini rematados igualmente por sendas rosetas de cinco pétalos y botón central, de mayor tamaño, unidas todas ellas por dos elegantes espirales. En el frontal está decorada en relieve con elementos arquitectónicos, concretamente dos bucráneos y una guirnalda de hojas de laurel (sertum), la altura de los bucráneos es de $16 \mathrm{~cm}$ y el sertum tiene una anchura máxima entre los dos extremos superiores de $17 \mathrm{~cm}$ y una altura en su parte central de $10 \mathrm{~cm}$; en los costados tiene sendos 'árboles de la vida' de $43 \mathrm{~cm}$ de altura y en la parte posterior una corona de $5 \mathrm{~cm}$ de grosor y $26 \mathrm{~cm}$ de diámetro tomando como

\footnotetext{
${ }^{34}$ CIL II 1325; ILER 2802; IRPC 14.
} 


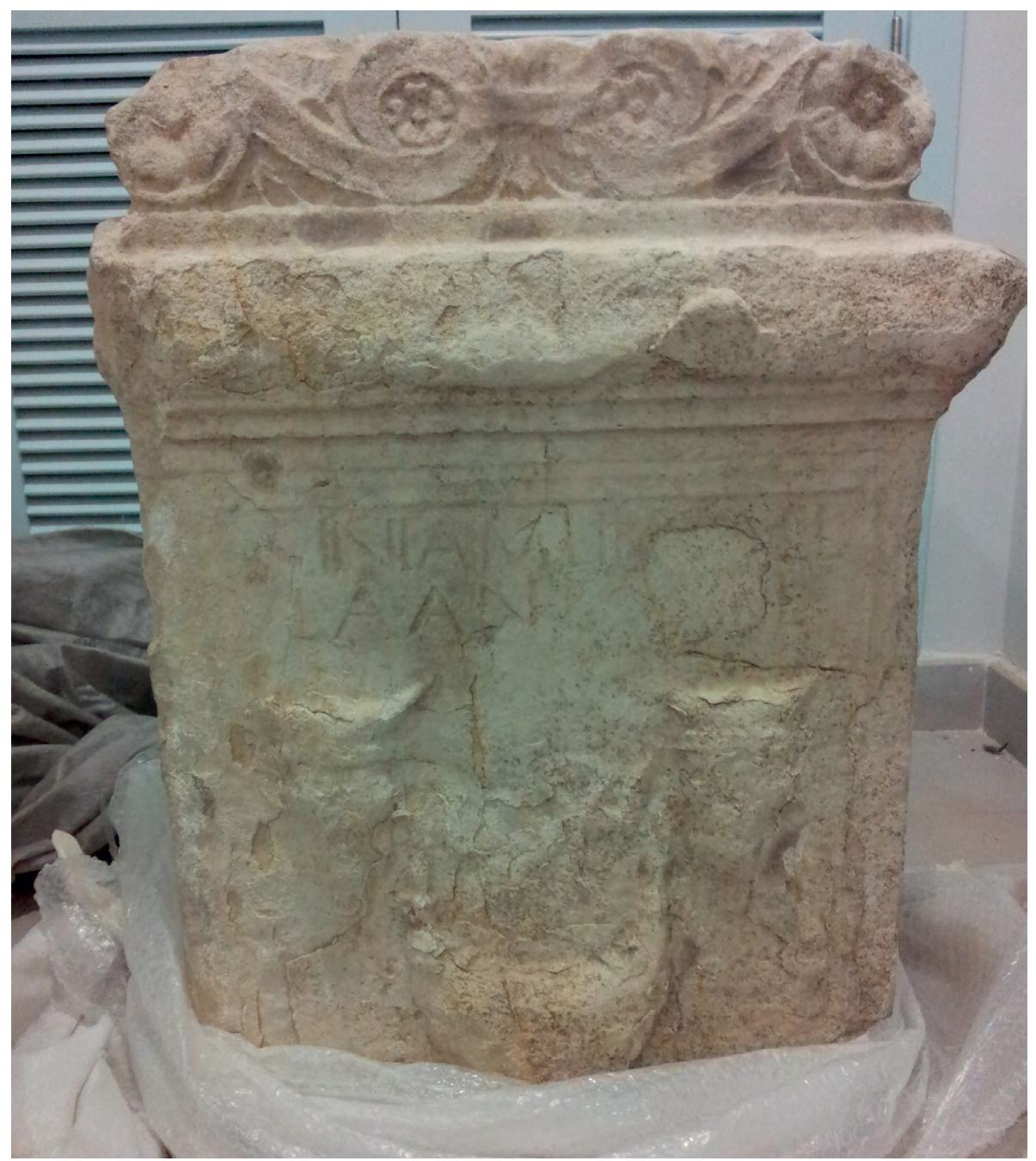

Figura 7. Ara de Licinia Gallula.

referencia los puntos exteriores. Mide $70 \mathrm{~cm}$ de altura, $48 \mathrm{~cm}$ de anchura y $35 \mathrm{~cm}$ de grosor; la altura de las letras oscila entre 3-3,4 cm. Se encontraba empotrada en una esquina del exterior de la capilla mayor del convento de San Agustín de Medina Sidonia, de donde ha sido trasladada recientemente al Museo Arqueológico de Medina, donde se encuentra en la actualidad. Su texto es el siguiente:

\section{LICINIA.M.LIB.GALL-}

VLA.AN.L.S.T.T.L

"Licinia Gálula, liberta de Marco, de 50 años. Sea para ti la tierra leve". 
CIL II 1327; Romero de Torres, op.cit. 216; ILER 2635, 5024; IRPC 16.

Las letras son capitales cuadradas, de elegante dibujo, el travesaño de la A centrado, los ángulos de la $\mathrm{N}$ y $\mathrm{M}$ formados en el vértice, la $\mathrm{C}$ semicircular, etc. El texto estaba, al parecer, escrito sobre una capa de mortero, no sobre la misma piedra, lo que ha contribuido en cierto sentido a la pérdida de algunas letras del texto, que hace algún tiempo aún podía leerse por completo.

Los Licinii son el sexto gentilicio más frecuente en Hispania con más de 300 testimonios, de ellos diez en la provincia de Cádiz. En cambio, Gallula, diminutivo afectivo formado mediante el sufijo - ulla, 'la pequeña Gala', está atestiguado solamente en una inscripción de Cádiz (CIL II 1912) y es desconocido entre los cognomina latinos ${ }^{35}$.

La existencia de este ara formando parte de la edificación, al igual que un fuste acanalado y un capitel corintio, ambos de mármol, que se encontraban en el mismo emplazamiento de la fachada lateral de San Agustín, pueden tener su explicación en el hecho de que en 1575, cuando vinieron a fundar su convento los Agustinos Calzados, el duque de Medina Sidonia les regaló el material que tenía acopiado para la obra de su palacio, que sin duda en buena medida provendría de los terrenos extramuros, donde en esos momentos todavía afloraban las ruinas de la ciudad romana ${ }^{36}$.

Fecha: segunda mitad del siglo I d.C. o primera del II d.C, según el tipo de letra y la rica ornamentación del ara.

\section{FRAGMENTO DE INSCRIPCIÓN}

Placa de mármol blanco que ha perdido todos sus márgenes y adopta una forma triangular con el ángulo a la izquierda; mide $8,7 \mathrm{~cm}$ de altura máxima, $9,6 \mathrm{~cm}$ el lado superior, $8,8 \mathrm{~cm}$ el lado inferior y $2,5 \mathrm{~cm}$ de grosor; sólo ha conservado una letra completa (de $2,7 \mathrm{~cm}$ de altura) y restos de ellas en las 11. 1-2; fue encontrada descontextualizada en el castillo de Medina Sidonia ( $3^{a}$ fase de excavación, año 2012). Su texto es el siguiente:

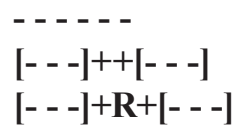

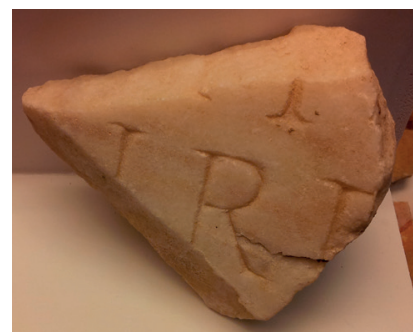

Figura 8. Fragmento inscripción.

\footnotetext{
${ }^{35}$ Cfr. I. Kajanto, The Latin Cognomina (Helsinki 1965) 196 tan sólo recoge este ejemplo de Asido.

${ }^{36}$ Cfr. F. Martínez y Delgado, Historia de la ciudad de Medina Sidonia (Cádiz 1875) 209.
} 
Las letras son capitales cuadradas, de grabado profundo, la $\mathrm{R}$ tiene el ojo cerrado y el trazo inclinado se prolonga a lo largo de la línea de caja.

Fecha: podríamos fecharla, según el tipo de letra, en la segunda mitad del siglo II d.C.

\section{Grafito}

Fragmento de placa o losa de barro de color beige-verdoso, que mide en la parte superior $15,5 \mathrm{~cm}, 5,7 \mathrm{~cm}$ el lateral izquierdo, $17 \mathrm{~cm}$ en la base y $14,5 \mathrm{~cm}$ el lateral derecho; este último es el único margen original que se conserva, si bien con algunas roturas, tiene dos líneas de texto, la segunda completa; al cortarse la primera línea de texto en el margen derecho, lo que falta de la inscripción se prolongaría por una losa adyacente. La altura de las letras oscila entre los 3 y $3,5 \mathrm{~cm}$ (1. 1) y los 3,5 y 4 (1. 2). Hallazgo casual producido en zona de Bellavista, ladera Oeste del Cerro de Medina Sidonia, margen exterior del núcleo urbano, también del de la ciudad romana. En las inmediaciones se han detectado importantes bolsas de materiales romanos, que pudiera interpretarse por su situación como vertedero o basurero, la misma zona de la terra sigillata con el grafito (cfr. infra ins. 11). En la actualidad se encuentra depositado en el Museo Arqueológico de Medina Sidonia. Su texto es el siguiente:

\section{AEFLANTI VERPA}

Las letras son capitales cursivas, propias de los grafitos parietales, el trazo central de la A es vertical sin tocar las barras transversales, la $\mathrm{E}$ y la $\mathrm{F}$ están representadas por dos líneas verticales, la segunda de la $\mathrm{F}$

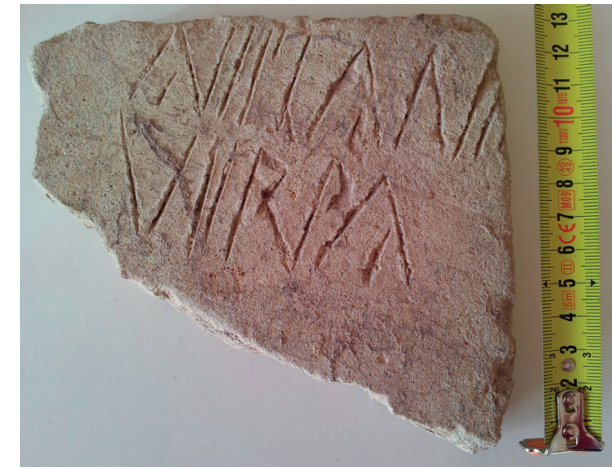

Figura 9. Inscripción de Verpa. más corta, los ojos de la P y R abiertos, y el travesaño horizontal de la L se inclina hacia abajo. Al final de la 1.1 se conservan dos trazos verticales, el primero creemos que corresponde a la $t$ del sufijo $-n t$, y el segundo a una $-i$.

La interpretación del texto resulta compleja. Se trata evidentemente de un mensaje erótico muy frecuente en los grafitos, del que la epigrafía de Pompeya nos ha ofrecido numerosos ejemplos. En efecto, uerpa significa 'pene', y puede considerarse en realidad como una variante de mentula, aunque se emplea con un tono claramente ofensivo y burlesco, de claro tinte popular, según se deduce de su empleo en las fuentes literarias: Catulo (28.12), Marcial (11.46.2), el Corpus Priapeorum (34.5), y sobre todo en los graffiti pompeyanos, donde son numerosos. La 
comparación de los testimonios literarios y epigráficos nos permite deducir que con el vocablo uerpa se quiere indicar un prepucio hacia atrás como resultado de una erección o incluso una actividad sexual excesiva. Incluso a menudo vemos cómo se emplea uerpa para indicar un agresivo acto homosexual, así en Catull. 28.12 (nam nihilo minore uerpa / farti estis?) y en CIL IV, 2360 encontramos una alusión metafórica a una irrumatio, y los graffiti pompeyanos parecen señalar a un irrumator o un pedicator ${ }^{37}$.

La interpretación de la 1. 1 resulta muy difícil, sobre todo porque ignoramos si había alguna línea más en la parte perdida. En efecto, por un lado, el comienzo AEFL - no está documentado en la lengua latina y, por otro, el sufijo - nt- indica que se trata de un participio de presente, que ha de ser necesariamente de efflare 'emitir, exudar', con simplificación de geminadas, o de flare 'soplar'. Al tratarse de una actividad realizada por la uerpa, el significado de efflare resulta mucho más acertado, por lo que pensamos que tendríamos una representación de un falo en el momento de eyacular, según ejemplos bien conocidos de Pompeya.

En cuanto a la $A$-inicial deberá ser o bien el final de una palabra perdida (no lo parece), con lo que tendríamos un ablativo ef(f)lanti uerpa, o bien, lo que nos parece más probable en este contexto, la interjección $a$, lo que implicaría un acusativo exclamativo con pérdida de la $-m$ final, fenomeno fonético muy frecuente en latín arcaico y vulgar ${ }^{38}$. Así, pues, proponemos la siguiente restitución, aunque comprendemos que se trata de una simple hipótesis de trabajo:

¡Mira, un pene eyaculando!

Fecha: segunda mitad del siglo I a.C. o primeros decenios del I d.C., según el tipo de letra y los fenómenos fonéticos que hemos señalado.

\section{Grafito SOBRe terRa Sigillata}

Fragmento de vaso o cuenco de terra sigillata, probablemente hispana por la calidad del barniz, de pasta anaranjada y barniz marrón-rojizo, con una inscripción en el interior del recipiente, cerca del fondo, realizado tras la fabricación, con incisiones poco profundas hechas con punzón fino; de forma irregular, mide $2,5 \mathrm{~cm}$ de altura, $2,8 \mathrm{~cm}$ de anchura máxima (coincide más o menos con la longitud de la inscripción), y $0,5 \mathrm{~cm}$ de grosor; el pie conservado del vaso mide $0,8 \mathrm{~cm}$ de altura; la altura de las letras oscila entre los $3 \mathrm{~mm}$ de la $\mathrm{N}$ y $\operatorname{los} 7 \mathrm{~mm}$ de la $\mathrm{T}$. Fue encontrado hace ya algunos años de forma casual en la zona de Bellavista, ladera Oeste del Cerro de Medina Sidonia, margen exterior del núcleo urbano de la ciudad romana, en cuyas inmediaciones se han detectado importantes bolsas de materiales romanos, lugar que pudiera interpretarse por su situación como

\footnotetext{
${ }^{37}$ J. N. Adams, The Latin Sexual Vocabulary (Maryland-Baltimore 1982) 14.

${ }^{38}$ Cfr. Bassols, op. cit. 190.
} 
vertedero o basurero. Fue depositado en el Museo Arqueológico de Medina por el propietario de los terrenos, donde se encuentra en la actualidad. El texto del grafito es el siguiente:

\section{ARETIN}

Las letras son desiguales y toscas de ejecución, el trazo horizontal de la A se abre en un ángulo hacia abajo, el ojo de la $\mathrm{R}$ abierto, los trazos de la E iguales, etc. Al final hay una

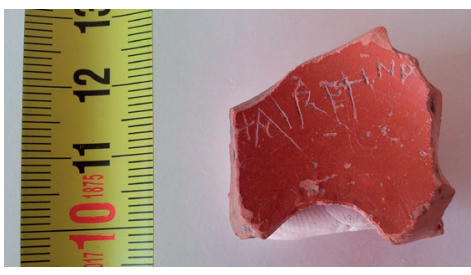

Figura 10. Grafito de Aretinus. letra de difícil interpretación, pues puede ser una $\mathrm{O}$ o una $\mathrm{V}$ donde esperaríamos una $\mathrm{I}$, por lo que tal vez se trate de un signo de interpunción. Delante del texto hay un signo en forma de X inclinada, aunque pudiera tratarse de una T, abreviatura del praenomen. En el plano gramatical hay que hablar de una simplificación de geminadas, ya que el nombre de la ciudad de la que procede es Arretium; en cualquier caso, no está atestiguado en la epigrafía hispana, e incluso Kajanto tan sólo recoge dos testimonios, uno de ellos en la propia ciudad de Arretium $^{39}$.

La datación resulta muy difícil, tal vez siglo I d.C.

\section{Grafito sobre Campaniense B}

Fragmento de plato en cerámica Campaniense B con una inscripción realizada tras la fabricación de la pieza, en la base exterior, sobre superficie no recubierta del barniz negro, que mide (tomando como referencia la superficie con inscripción): $5,5 \mathrm{~cm}$. lado izquierdo, $7,5 \mathrm{~cm}$ lado derecho, 8,5 lado inferior; grosor medio: $0,9 \mathrm{~cm}$; la altura de las letras es de $9 \mathrm{~mm}$. Tiene estampillas en el perímetro interno del fondo de la pieza, de $7 \times 7$ $\mathrm{mm}$. Fue encontrada en el castillo de Medina Sidonia, en la $2^{\mathrm{a}}$ fase de las excavaciones en extensión, realizadas en la campaña del 2010-2011 en el estrato republicano romano, asociado a la base interior de la muralla Este del castellum. Tiene grabado con punzón el siguiente grafito:

\section{M(arci) FLA[VI ---]}

“De Marco Flavio...".

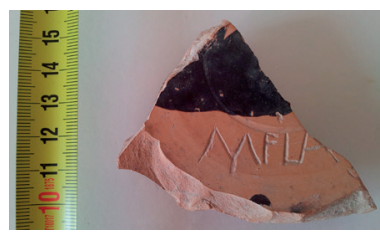

Figura 11a. Grafito de M. Flavi.



Figura 11b. Campaniense B

${ }^{39}$ Kajanto, op. cit. 58, 119. 
A pesar de ser un grafito, las letras son capitales cuadradas de grabado profundo y elegante ejecución; así, por ejemplo, la M tiene los trazos paralelos, el inferior de la L es recto, la A es abierta y con el trazo horizontal recto, el inferior de la $\mathrm{F}$ algo más corto y cercano al superior.

Este epígrafe resulta interesante por la presencia en época republicana de un individuo perteneciente a la gens Flauia en Hispania, siendo sin duda la primera referencia de un ciudadano romano o latino en Asido. Por otra parte, el gentilicio Flavio está atestiguado en Asido en el epígrafe funerario de Flauia Quinti$l l a^{40}$. La fecha más probable de esta pieza es finales del siglo II a.C. o primera mitad del I a.C. 